\title{
Simplified Theodolite Calibration for Robot Metrology
}

\author{
Ibrahim A. Sultan \\ School of Science and Engineering \\ University of Ballarat \\ PO Box 663 \\ Ballarat VIC 3353 \\ Australia \\ John G. Wager \\ Department of Mechanical and Materials Engineering \\ The University of Western Australia \\ Nedlands WA 6907 \\ Australia
}

Keywords: $\quad$ Calibration - Robot - Theodolite - Kinematic Model - Epipolar

\begin{abstract}
$\underline{\text { Abstract: }}$
Theodolites represent a well-established 3D-point-measuring technology. However when used for robot applications they have to be properly calibrated to fulfil the necessary accuracy requirements. The theodolite calibration methods, which have been reported in the literature, involve the use of costly sophisticated equipment not easily available to most users. Therefore, a new simplified calibration technique is presented based on the use of a graduated precision bar suspended freely to align with the vertical direction.
\end{abstract}

To develop efficient mathematical models, the theodolites will be regarded as $2 \mathrm{R}$ open-ended mechanisms with the end-effector axis directed along the line of sight. The proposed models are then coded in a computer program designed to verify the validity of the technique presented. The simulation results will be presented at the end of the paper. 


\section{Introduction and Literature Survey:}

It is essential for robot calibration to precisely measure the spatial characteristics of the end-effector at many locations and compare the measured quantities with their corresponding nominal values using a suitable mathematical procedure. To acquire the necessary spatial measurements, researchers have implemented different approaches that range from simple conventional contact methods such as dial indicators and mechanical fixtures to costly automated laser-tracking systems.

Some factors should be examined before any particular three-dimensional (3D) measuring system is decided upon. The first factor to consider here would be the level of precision desired for the collected data and how it compares with the established precision of the proposed measuring approach. This is particularly important because the improved positioning accuracy of the calibrated robot manipulator is limited by the accuracy of the measurement system employed.

Economical viability of the measuring system as weighed against the expected gain of the calibration work is another factor to consider during the selection process. Measuring systems vary in cost according to the level of automation, precision and operating skills involved. Also the time required to collect the data might be a factor to consider in some applications, especially in production environment where the robot has already been in operation and the calibration process is only a part of a scheduled maintenance routine. In such a case, a low-cost, simply built and readily used system may be a preferred option. The system, which was designed by Everett and Ives (1996), is a representative example in this regard. This short-range system 
uses LED beam trip switches to define the spatial location of a sphere attached to the end-effector.

Generally, measuring systems may be broadly classified according to the amount of spatial data they return per one measurement. Usually, six parameters are needed to locate a solid object in space. Three of these parameters may represent the XYZ Cartesian location of any point on the object whilst the other three parameters are intended to report the angular orientation of the object. For robot calibration, it is preferable that the measuring system employed is able to return all the six spatial characteristics of the end-effector and therefore produce full-pose measurements. Even though it is long established that the spatial measurement of angles is a difficult task to achieve, a system capable of generating complete, six parametric, measurements was reported by Vincze et al (1994). The system uses a single laser beam to measure the Cartesian location of a target attached to the end-effector while the orientation is determined by analysing the intensity profile of the reflected beam. The system, which is fully automated, is able to track and measure random movements of the robot in space. Other laser tracking systems were reported by Van Brussel (1990) and Nakamura et al (1994). These systems however seem to produce only partial-pose measurements of the end-effector spatial locations. This is in fact the case with the overwhelming majority of the measuring systems already in existence. They mostly generate information related to the spatial position of a point attached to the end-effector. The automated theodolite system designed by Driels and Pathre (1991) is a good example of these systems. This system is different in the sense that it uses a charge-coupled device (CCD) and an image-analysis technique to track an illuminated spot attached to the end-effector. A CCD camera is mounted on 
a stepper-motor-driven, two revolute-joint mechanism similar to that used on theodolites to facilitate the tracking performance. A single CCD camera system was also successfully used by Preising and Hsia (1991) to calibrate a robot arm. In this system, the image of 36 infinitesimal disks of known dimensions, inscribed on a plate attached to end-effector, was analysed to calculate the required spatial information.

Point measuring systems are widely used for robot calibration where researchers may use only the spatial positions of a measured point attached to an end-effector in a mathematical procedure to compute the corresponding values of the geometric parameters of the robotic structures. Some of these systems use laser interferometry to measure positioning errors along one of the axes of a given Cartesian frame. Examples of these systems are presented by Tang and Liu (1993) and Legnani et al (1996), where the robot is made to move along linear paths in the direction of either the $\mathrm{Y}$ - or $\mathrm{X}$-axis, parallel to a laser beam, and linear errors are measured.

A more generalised point measuring technique is achieved through the use of coordinate measuring machines (CMM). These are mostly built out of three prismatic-joints where the joint-axes are directed along the three axes of a Cartesian frame. The Cartesian location of any target located within the machine work space will be displayed once it comes in contact with a probe. Mooring et al (1991) present a good example of these systems where the positioning errors of a PUMA-type robot are directly measured.

The method of triangulation is often used to measure the spatial locations of points. In this method, two, or more, lines are made to intersect at the point whose spatial 
position is required to be measured. The spatial particulars of these lines, with respect to a defined frame, are measured and used in a mathematical procedure to calculate the position of the observed point with respect to a known frame. One approach was reported by Stone and Sanderson (1987), where triangulation is achieved by emitting sound pulses from a source attached to the end-effector and the time taken by the sound wave to travel to microphones located at known positions, was used to work out the distances from the source to the microphones. These distances are then used in a triangulation procedure to work out the position of the end-effector in space. This method is indeed automatic and fast but sensitive to changes in atmospheric conditions and therefore prone to errors as discussed by Mooring et al (1991).

Theodolites are also employed to measure the spatial position of a target attached to the end-effector. The lines of sight of two, or more, theodolites are used to achieve the required triangulation. This technique was used by Judd and Knasinski (1990) to successfully calibrate an Automatix AID-900 robot. There is no mention in this work as to how the two theodolites were calibrated and consequently how the kinematic relations between them were established. Whitney et al (1986) used a single theodolite and a bar with a predefined length to calibrate a PUMA-type robot. In this work there is no reference to the method used to calibrate the measuring theodolite.

Jarvis (1988) notes that the simple geometry used to describe the kinematics of theodolites in surveying applications is not sufficient for robot calibration purposes. Jarvis therefore proposes a technique where every theodolite is calibrated individually by observing a target, moving along a straight line, as it pauses at a set of defined points. The target is mounted on a robotic arm and the distances between the 
measurement points are determined by a laser interferometric system. The kinematic relations between two theodolites are then established by mutual observation of spatial targets located within the work volume. The sophisticated equipment used to calibrate theodolites in this work made it possible to use the absolute, rather than relative, location of the observed point in the mathematical model. However such a level of sophistication is rarely possible to attain in an industrial situation and as such defeats the purpose of simplicity for which theodolites are used for robot calibration. The procedure is also prone to error accumulation which may result from both positioning errors of the robotic arm and the laser interferometer. The method results in theodolites calibrated only in the narrow portion of the work volume which is relevant to the straight line along which the target is moved.

Driels and Pathre(1991) also calibrated a single theodolite, which was built to carry a CCD camera. In this work a CMM was employed to calibrate the theodolite in a limited portion of the work volume using the kinematic notation described by Hayati (1983).

This paper reports a proposed procedure for the calibration of a two-theodolite module. The procedure, which is referred to as the Vertical-Observation-Lines method, involves the use of a published kinematic notation referred to as the $\phi$-model. The main aspects of this notation is described in the attached appendix, but more details may be sough in a work Sultan and Wager (1999). The same notation was also used by Sultan and Wager (2001) successfully to calibrate an industrial six-axes robot. 


\section{The Method of Vertical-Observation-Lines:}

In the present work the theodolites were made to observe two points, $\mathrm{P}^{\mathrm{i} 1}$ and $\mathrm{P}^{\mathrm{i} 2}$ whose position vectors in a base Cartesian frame are, $\mathbf{p}^{i 1}$ and $\mathbf{p}^{i 2}$ respectively. The two points are separated by a known distance, $l^{i}$, along a line parallel to the vertical axis of the base frame. This line will be referred to, in the following discussion, as the vertical-observation-line or VO-line for short. In other words, the two observed points are made to share both the $\mathrm{x}$ - and $\mathrm{y}$-coordinates and their relative position vector, $\mathbf{l}^{i}$, is fully defined, in a base Cartesian frame, as follows;

$\mathbf{l}^{i}=0 \mathbf{x}+0 \mathbf{y}+l^{i} \mathbf{z}$

where $\mathbf{x}, \mathbf{y}$ and $\mathbf{z}$ are unit vectors parallel to the corresponding axes of the base frame and the length, $l^{i}$, is accurately measured.

The same position vector when calculated as observed by the theodolites using their erroneous geometric parameters and then transformed to the base frame is referred to in this discussion as $\Delta \mathbf{p}^{i}$. This vector can be expressed as follows;

$\Delta \mathbf{p}^{i}=\Delta p_{x}^{i} \mathbf{x}+\Delta p_{y}^{i} \mathbf{y}+\Delta p_{z}^{i} \mathbf{z}$

where $\Delta p_{x}^{i}, \Delta p_{y}^{i}$ and $\Delta p_{z}^{i}$ are the $\mathrm{X}$-, $\mathrm{Y}$ - and Z-components respectively of the position vector $\Delta \mathbf{p}^{i}$. The scalar quantities, $\Delta p_{x}^{i}, \Delta p_{y}^{i}$ and $\Delta p_{z}^{i}$ are expressed in functional forms as follows;

$\Delta p_{x}^{i}=f_{x}^{i}\left(\boldsymbol{\theta}^{i}, \mathbf{q}\right), \quad \Delta p_{y}^{i}=f_{y}^{i}\left(\boldsymbol{\theta}^{i}, \mathbf{q}\right)$ and $\quad \Delta p_{z}^{i}=f_{z}^{i}\left(\boldsymbol{\theta}^{i}, \mathbf{q}\right)$

where $\boldsymbol{\theta}^{i}$, is a vector of the eight theodolite-angles (obtained from observations) which correspond to VO-line number $i$ and $\mathbf{q}$ is a vector encompassing the system's 21 geometric parameters. These 21 parameters will be detailed in section (3) below. 
The VO-lines method is useful in the formulation of the mathematical model, because it provides three equations, instead of the common one length equation, per two observations. Each equation describes the relative error in a direction parallel to one of the three perpendicular axes of the base frame. Two more equations can also be written per a VO-line to describe the kinematic consistency of the observation process. This will bring the number of useful equations per line to five; hence decreasing the number of observations required for the calibration process.

In the present work, VO-lines with different lengths and altitudes would be moved around in the work volume from one location to the other and at each location, $i$, two points separated by different lengths, $l^{i}$, are observed. Therefore; the following error equation can be written at every location of the VO-line;

$\mathrm{e}^{i}=\Delta \mathrm{p}^{i}-\mathbf{l}^{i}$

where $\mathbf{e}^{i}$ is the dimensional error vector associated with the VO-line number $i$ in directions parallel to the corresponding axes of the base frame.

After the VO-line was moved through an adequate number of locations distributed around the work volume resulting in the compilation of an adequate number of error equations, a suitable least-squares technique may be implemented for the mathematical realisation of the procedure. The models proposed here for theodolite calibration are presented in the next section. 


\section{Mathematical Procedure:}

A two-theodolite module is shown in figure (1). The figure also shows the VO-line number $i$ with the observation target points $\mathrm{P}^{i 1}$ and $\mathrm{P}^{i 2}$ separated by a distance $l_{i}$. In the present discussion, spatial characteristics of the left-hand side (LHS) theodolite are designated by the subscript $\mathrm{L}$ while the subscript $\mathrm{R}$ is used for the spatial characteristics of the right-hand side (RHS) theodolite.

The $\phi$-model frames are attached to the links of both theodolites as shown in figure

(1). The frames are assigned according to the conventions presented in the appendix as follows;

1. $\mathrm{X}_{0} \mathrm{Y}_{0} \mathrm{Z}_{0}$ is the base frame.

2. $\mathrm{X}_{L 1} \mathrm{Y}_{L 1} \mathrm{Z}_{L 1}$ is constructed about the near-vertical joint-axis of the LHS theodolite and this frame will be referred to as the L1-frame.

3. $\mathrm{X}_{L 2} \mathrm{Y}_{L 2} \mathrm{Z}_{L 2}$ is constructed about the near-horizontal joint-axis of the LHS theodolite and it will be referred to as the L2-frame.

4. $\mathrm{X}_{L} \mathrm{Y}_{L} \mathrm{Z}_{L}$ is constructed about the line of sight of the LHS theodolite. This frame is referred to, here, as the L-frame.

5. $\mathrm{X}_{R 1} \mathrm{Y}_{R 1} \mathrm{Z}_{R 1}$ is constructed about the near-vertical joint-axis of the RHS theodolite and will be referred to as the R1-frame.

6. $\mathrm{X}_{R 2} \mathrm{Y}_{R 2} \mathrm{Z}_{R 2}$ is constructed about the near-horizontal joint-axis of the RHS theodolite and this frame will be referred to as the R2-frame.

7. $\mathrm{X}_{R} \mathrm{Y}_{R} \mathrm{Z}_{R}$ is constructed about the line of sight of the RHS theodolite and is referred to, in the present discussion, as the R-frame. 
It is worth noting here that the z-axis of the base frame is made to coincide with the absolute vertical direction and intersect the z-axis of the L1-frame. The point of intersection is the origin of both the base frame and the L1-frame. Moreover, the $y$ axis of the base frame is made to intersect the z-axis of the R1-frame. These frames are related, according to the conventions of the $\phi$-model, by the following sets of 21parameters;

$\left\{a_{R 2}, b_{R 2}, \alpha_{R 2}\right.$ and $\left.\beta_{R 2}\right\}$ relate the R-frame to the R2-frame, $\left\{a_{R 1}, b_{R 1}, \alpha_{R 1}\right.$ and $\left.\beta_{R 1}\right\}$ relate the R2-frame to the R1-frame, $\left\{a_{R 0}, \alpha_{R 0}\right.$ and $\left.\beta_{R 0}\right\}$ relate the R1-frame to the base-frame, $\left\{a_{L 2}, b_{L 2}, \alpha_{L 2}\right.$ and $\left.\beta_{L 2}\right\}$ relate the L-frame to the L2-frame, $\left\{a_{L 1}, b_{L 1}, \alpha_{L 1}\right.$ and $\left.\beta_{L 1}\right\}$ relate the L2-frame to the L1-frame and $\left\{\alpha_{0}\right.$ and $\left.\beta_{0}\right\}$ relate the L1-frame to the base frame.

The values of $\phi_{i}$-angles are selected as follows;

$\phi_{L 2}=0.0, \phi_{L 1}=0.0, \phi_{R 2}=0.0, \phi_{R 1}=0.0, \phi_{0}=\pi / 2$.

Figure (1) depicts the instant when the two theodolites are observing point, $\mathrm{P}^{i j}$, number $j$ (where $j=1$ or 2 ) on the VO-line number $i$. At this instant, the lengths of the lines of sight of the LHS-theodolite and the RHS-theodolite are $h_{L}^{i j}$ and $h_{R}^{i j}$ respectively. The spatial location of the observed point with respect to the L-frame, $\mathbf{p}_{L}^{i j}$, can be expressed as follows;

$\mathbf{p}_{L}^{i j}=0 \cdot \mathbf{x}_{L}^{i j}+0 \cdot \mathbf{y}_{L}^{i j}+h_{L}^{i j} \mathbf{z}_{L}^{i j}$

where $\mathbf{x}_{L}^{i j}, \mathbf{y}_{L}^{i j}$ and $\mathbf{z}_{L}^{i j}$ are unit vectors directed along the axes of the L-frame. 
The position vector of the same point with respect to the R-frame, $\mathbf{p}_{R}^{i j}$, can be written as follows;

$\mathbf{p}_{R}^{i j}=0 . \mathbf{x}_{R}^{i j}+0 . \mathbf{y}_{R}^{i j}+h_{R}^{i j} \mathbf{z}_{R}^{i j}$

where $\mathbf{x}_{R}^{i j}, \mathbf{y}_{R}^{i j}$ and $\mathbf{z}_{R}^{i j}$ are unit vectors directed along the axes of the R-frame.

The technique of homogeneous transformation is used here to express both the position vectors in equations (5) and (6) with respect to the base-frame. In this case, the transformed position vectors can be equated as follows;

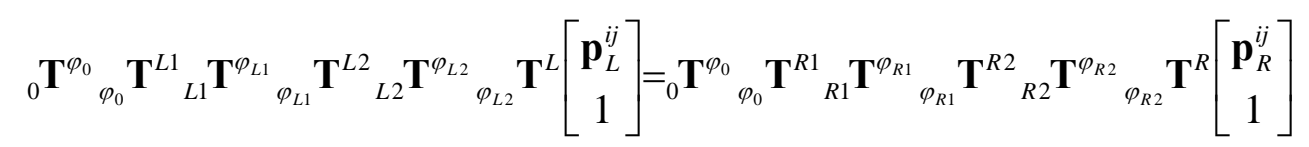

where the $\mathbf{T}$-matrices are constructed as outlined in the appendix. These matrices encompass the model parameters together with the joint-displacements of theodolites.

Substituting for $\mathbf{p}_{L}^{i j}$ and $\mathbf{p}_{R}^{i j}$ in equation (7) and performing the due matrix multiplication produces the following vector relation;

$h_{R}^{i j} \cdot \mathbf{z}_{R_{0}}^{i j}-h_{L}^{i j} \cdot \mathbf{z}_{L_{0}}^{i j}=\mathbf{p}_{L_{0}}^{i j}-\mathbf{p}_{R_{0}}^{i j}$

where $\mathbf{z}_{R_{0}}^{i j}$ and $\mathbf{z}_{L_{0}}^{i j}$ are unit vectors directed along the Z-axes of the R-frame and the L-frame respectively as expressed with respect to the base-frame. The position vectors, $\mathbf{p}_{R_{0}}^{i j}$ and $\mathbf{p}_{L_{0}}^{i j}$, locate the origins of the R-frame and the L-frame respectively with respect to the base-frame. 
The following system of linear equations, which relates to point number $i j$, can be worked out from equation (8);

$\mathbf{Z}_{0}^{i j}\left[\begin{array}{c}h_{R}^{i j} \\ h_{L}^{i j}\end{array}\right]=\Delta \mathbf{p}_{0}^{i j}$

where $\mathbf{Z}_{0}^{i j}$ and $\Delta \mathbf{p}_{0}^{i j}$ take the following forms;

$\mathbf{Z}_{0}^{i j}=\left[\begin{array}{ll}\mathbf{z}_{R_{0}}^{i j} & -\mathbf{z}_{L_{0}}^{i j}\end{array}\right]_{3 \times 2}$

and

$\Delta \mathbf{p}_{0}^{i j}=\left[\mathbf{p}_{L_{0}}^{i j}-\mathbf{p}_{R_{0}}^{i j}\right]_{3 \times 1}$

The values of $h_{L}^{i j}$ and $h_{R}^{i j}$ could now be worked out by applying a least-squares technique to equation (9) as follows;

$\left[\begin{array}{c}h_{R}^{i j} \\ h_{L}^{i j}\end{array}\right]=\left(\mathbf{Z}_{0}^{i j} \mathbf{Z}_{0}^{i j}\right)^{-1} \mathbf{Z}_{0}^{i j} \Delta \mathbf{p}_{0}^{i j}$

Equation (12) yields the following expression for $h_{L}^{i j}$;

$h_{L}^{i j}=\left(\frac{1}{1-\left(\mathbf{z}_{L_{0}}^{i j} \circ \mathbf{z}_{R_{0}}^{i j}\right)^{2}}\right)\left\{\Delta \mathbf{z}_{0}^{i j} \circ \Delta \mathbf{p}_{0}^{i j}\right\}$

where $\Delta \mathbf{z}_{0}^{i j}$ is given as follows;

$\Delta \mathbf{z}_{0}^{i j}=\mathbf{z}_{R_{0}}^{i j}\left(\mathbf{z}_{R_{0}}^{i j} \circ \mathbf{z}_{L_{0}}^{i j}\right)-\mathbf{z}_{L_{0}}^{i j}$

Once the length, $h_{L}^{i j}$ is calculated; the position vector, $\mathbf{p}_{0}^{i j}$, which relates the spatial location of the observed point with respect to the base-frame could be worked out as follows;

$\mathbf{p}_{0}^{i j}=\mathbf{p}_{L_{0}}^{i j}+h_{L}^{i j} \cdot \mathbf{z}_{L_{0}}^{i j}$ 
The differential form of equation (15) may be expressed as follows;

$$
\frac{\partial \mathbf{p}_{0}^{i j}}{\partial q_{k}}=\frac{\partial \mathbf{p}_{L_{0}}^{i j}}{\partial q_{k}}+h_{L}^{i j} \cdot \frac{\partial \mathbf{z}_{L_{0}}^{i j}}{\partial q_{k}}+\mathbf{z}_{L_{0}}^{i j} \frac{\partial h_{L}^{i j}}{\partial q_{k}}
$$

where $q_{k}$ refers to the geometric parameter number $k$ and,

$$
\frac{\partial \mathbf{h}_{L}^{i j}}{\partial q_{k}}=\left(\frac{1}{1-\left(\mathbf{z}_{L_{0}}^{i j} \circ \mathbf{z}_{R_{0}}^{i j}\right)^{2}}\right)\left\{\Delta \mathbf{z}_{0}^{i j} \frac{\partial \Delta \mathbf{p}_{0}^{i j}}{\partial q_{k}}+\Delta \mathbf{p}_{0}^{i j} \frac{\partial \Delta \mathbf{z}_{0}^{i j}}{\partial q_{k}}+2 h_{L}^{i j}\left(\mathbf{z}_{L_{0}}^{i j} \circ \mathbf{z}_{R_{0}}^{i j}\right) \frac{\partial\left(\mathbf{z}_{L_{0}}^{i j} \circ \mathbf{z}_{R_{0}}^{i j}\right)}{\partial q_{k}}\right\}
$$

The above concepts may now be applied to equation (4) to obtain a differential error form for the VO-line number $i$ as follows;

$$
\left(\mathbf{p}_{0}^{i 1}-\mathbf{p}_{0}^{i 2}\right)-\mathbf{l}^{i}=\sum_{k=1}^{21}\left(\frac{\partial \mathbf{p}_{0}^{i 1}}{\partial q_{k}}-\frac{\partial \mathbf{p}_{0}^{i 2}}{\partial q_{k}}\right) \delta q_{k}
$$

where $\mathbf{p}_{0}^{i 1}$ and $\mathbf{p}_{0}^{i 2}$ are calculated using equation (15) and the numerical values of the parameters as obtained in the previous iteration. The differential vectors $\frac{\partial \mathbf{p}_{0}^{i 1}}{\partial q_{k}}$ and $\frac{\partial \mathbf{p}_{0}^{i 2}}{\partial q_{k}}$ are as given in equation (16).

Equation (18) produces three scalar error equations per a VO-line to use for the calibration analysis. Two more equations per line (i.e. a single equation for every observed point) can also be obtained and utilized for the analysis. These two equations are relevant to the kinematic consistency of the theodolite module as described in the next section. 


\section{Kinematic Consistency:}

The expressions in equations (7) and (8) indicate that both theodolites must indeed be observing the same point, number $i j$, in order to ensure the accuracy of measurements. To achieve that, a kinematic consistency index may be proposed and used for the analysis by re-writing equation (8) in the following linear form;

$$
\left[\begin{array}{lll}
\mathbf{z}_{R_{0}}^{i j} & -\mathbf{z}_{L_{0}}^{i j} & \mathbf{p}_{L_{0}}^{i j}-\mathbf{p}_{R_{0}}^{i j}
\end{array}\right]\left[\begin{array}{c}
h_{R}^{i j} \\
h_{L}^{i j} \\
1
\end{array}\right]=\mathbf{0}
$$

For equation (19) to have a solution, the determinant of the system matrix must be equal to zero. Geometrically, this means that the intersecting axes, $\mathbf{z}_{R_{0}}^{i j}$ and $\mathbf{z}_{L_{0}}^{i j}$ must fall in the same plane as the vector $\mathbf{p}_{L_{0}}^{i j}-\mathbf{p}_{R_{0}}^{i j}$. This is equivalent to the co-planarity constraint, which is utilised in the field of computer vision to establish the elements of the epipolar geometry. In this geometry, the lines linking the centres of the cameras must fall in one plane with the intersecting optical rays of the cameras. Since these optical rays are relevant to a pair of corresponding points on two images, geometric relationships can be used to construct the necessary transformation information. Adequate information on the epipolar geometry may be sought in textbooks by $\mathrm{Xu}$ and Zhang (1996) and by Hartley and Zissermann (2000) or in papers by Xu (1995), Zhang (1998) and Zissermann and Maybank (1993).

In the present work, the kinematic consistency is quantified by calculating the determinant of the matrix given in equation (19). This determinant is referred to in the following discussion as the Kinematic Consistency Index, KCI. As such, the value of $K C I^{i j}$, which is relevant to point $i j$, may be expressed as follows;

$$
K C I^{i j}=-\left(\mathbf{p}_{L_{0}}^{i j}-\mathbf{p}_{R_{0}}^{i j}\right) \circ\left(\mathbf{z}_{R_{0}}^{i j} \times \mathbf{z}_{L_{0}}^{i j}\right)
$$


Equation (20) can then be manipulated into the following from;

$K C I^{i j}=\mathbf{z}_{R_{0}}^{i j} \circ\left(\mathbf{p}_{L_{0}}^{i j} \times \mathbf{z}_{L_{0}}^{i j}\right)+\mathbf{z}_{L_{0}}^{i j} \circ\left(\mathbf{p}_{R_{0}}^{i j} \times \mathbf{z}_{R_{0}}^{i j}\right)$

This last expression (21) produces two extra equations (one for every observed point) to use for the VO-line. The differential form of $K C I^{i j}$ is expressed as follows;

$$
\frac{\partial K C I^{i j}}{\partial q_{k}}=\mathbf{z}_{R_{0}}^{i j} \circ \frac{\partial\left(\mathbf{p}_{L_{0}}^{i j} \times \mathbf{z}_{L_{0}}^{i j}\right)}{\partial q_{k}}+\frac{\partial \mathbf{z}_{R_{0}}^{i j}}{\partial q_{k}} \circ\left(\mathbf{p}_{L_{0}}^{i j} \times \mathbf{z}_{L_{0}}^{i j}\right)+\mathbf{z}_{L_{0}}^{i j} \circ \frac{\partial\left(\mathbf{p}_{R_{0}}^{i j} \times \mathbf{z}_{R_{0}}^{i j}\right)}{\partial q_{k}}+\frac{\partial \mathbf{z}_{L_{0}}^{i j}}{\partial q_{k}} \circ\left(\mathbf{p}_{R_{0}}^{i j} \times \mathbf{z}_{R_{0}}^{i j}\right)
$$

For model implementation, $K C I^{i j}$ is used as follows;

$$
\left(K C I^{i j}-0\right)=\sum_{k=1}^{21} \frac{\partial K C I^{i j}}{\partial q_{k}} \delta q_{k}
$$

where $K C I^{i j}$ is calculated numerically from equation (21) using the values obtained during the previous iteration for the system parameters and $\frac{\partial K C I^{i j}}{\partial q_{k}}$ is evaluated from equation (22).

After the data related to a total of $n$ VO-lines are collected and the corresponding aggregate $5 n \times 1$ error vector, e, is worked out, the overall error equation of the model may be expressed as follows;

$\mathbf{e}=\mathbf{J} \delta \mathbf{q}$

where $\mathbf{J}$ is the, $5 n \times 21$, aggregate Jacobian matrix of the model.

The solution of the over-determined system in equation (24) may be obtained by the use of a suitable least-squares technique as follows;

$\left(\mathbf{J}^{T} \mathbf{J}\right) \delta \mathbf{q}=\mathbf{J}^{T} \mathbf{e}$ 
The output of equation (25) is the vector of differential parameters, $\delta \mathbf{q}$. The iterations stop when the norm of this vector is less than or equal to a small predefined value. The values of the system parameters are updated for each iteration such that the vector of parameters, $\mathbf{q}^{r}$, which may be used in iteration number $r$ is worked out as follows;

$\mathbf{q}^{r}=\mathbf{q}^{r-1}-\delta \mathbf{q}^{r-1}$

where $\delta \mathbf{q}^{r-1}$ is the vector of differential parameters obtained at iteration number $r-1$.

The Levenberg-Marquardt technique can be implemented to solve the system given in equation (25) as follows;

$\left(\mathbf{J}^{T} \mathbf{J}+\lambda \mathbf{I}\right) \delta \mathbf{q}=\mathbf{J}^{T} \mathbf{e}$

where $\mathbf{I}$ is a $21 \times 21$ identity matrix and $\lambda$ is a non-negative coefficient selected in such a manner that the matrix $\left(\mathbf{J}^{T} \mathbf{J}+\lambda \mathbf{I}\right)$ is always positive definite.

In this technique, the user selects a suitable value for $\lambda$ and this value is gradually decreased as the solution converges to a minimum to retain the favourable convergence properties of Gauss-Newton method. Useful insights into this strategy are available in publications by Mooring et al (1991) and Marquardt (1963).

The system in equation (24) could also be solved by the use of a suitable Kalman filter technique, which involves a recursive stepwise estimation procedure. In this technique, which may be reviewed in Mooring et al (1991) or Bay (1993), the data collected for one VO-line may be used to estimate the system parameters. The values of these parameters would then be improved by the sequential use of the data 
collected in connection with other VO-lines one at a time. This method may be best suited for the autonomous, on-the-fly, calibration methods rather than aggregate batch data-collection techniques.

The mathematical procedure proposed in this section was performed, symbolically, on a computer algebra package and the resulting output was coded in a computer program. The input to the program consists of the theodolite angles that correspond to all VO-lines simulated in conjunction with the nominal ideal values of the model parameters.

The computer program was successfully used to obtain a calibrated model for a twotheodolite module. The results of the simulation work are given in the next section.

\section{Simulation and Results:}

The mathematical model presented in sections (3) and (4) was used in a computer program designed to simulate the calibration process of a two-theodolite module. The input to the program consists of the set of nominal geometric parameters in addition to the theodolite angles (i.e. $\boldsymbol{\theta}^{i}$ ) identifying the spatial positions of vertical linesattached points. These angles are, normally, obtained from observations.

The nominal values of the system geometric parameters are shown in table (1), where the angles are given in radians and lengths are in millimetres. The values in this table were obtained by assuming that the two theodolites would be erected and levelled at a distance 2400.0 from one another. Both lines-of-sight are assumed to be initially parallel to the $\mathrm{X}_{0}$ - axis and lying in the $\mathrm{xy}$-plane of the $\mathrm{X}_{0} \mathrm{Y}_{0} \mathrm{Z}_{0}$ - frame (i.e. the base 
frame). Without loss of generality, this configuration is meant to represent the home position (where all joint angles may be equated to zero) of the two-theodolite module.

The details of the calibration procedure are described in figure (2). The inverse kinematic procedure, pointed out in the figure, is performed to calculate the angular displacements of the theodolite joints in order for the line of sight to shoot at a point whose spatial coordinates are known. If the joint angles are given, then a direct kinematic procedure may be performed to calculate the spatial location of the observed point. The details of both the inverse and the direct kinematic procedures may be sought in works on robot kinematics; e.g. Sultan (2000).

As figure (2) indicates, small constant deviations were intentionally incorporated into the nominal values of the theodolite geometric parameters to simulate a real life situation. Table (2) shows the new values of these geometric parameters after small deviations were added.

Also, the angles obtained from observations were incremented by random errors introduced to simulate the effects of sensor resolutions. These errors were generated by a random function available in the C-compiler used for the simulation. A histogram representation of these errors is shown in figure (3), whereby the mean error and the standard deviations are given as 1.88 seconds and 17.59 seconds respectively.

Calibration procedure has been performed, as described in figure (2), and the resulting calibrated values for the theodolites' geometric parameters are given in table (3). A 
comparison between the values in this table and corresponding values in table (2) reveals that the deviations introduced initially to the geometric parameters differ slightly from the deviations in these parameters as obtained by the calibration procedure. This may be attributed to the effects of the sensor resolution errors, which were mapped into the calibration results.

To test the performance of the proposed theodolite calibration technique, a simulated set of predetermined vertical lengths were calculated using the geometric parameters of both uncalibrated (nominal) and calibrated theodolites. The details of this procedure are described in figure (4) and the x-, y- and z-errors obtained for both the calibrated and uncalibrated theodolites are shown in figures (5), (6) and (7) respectively. The mean errors in these directions have been reduced form 14.19 to $0.05 \mathrm{~mm}$, 9.7 to $0.024 \mathrm{~mm}$ and 10.3 to $0.044 \mathrm{~mm}$ respectively. Moreover, the corresponding standard deviations of error have been reduced respectively from 6.37 $\mathrm{mm}, 6.38 \mathrm{~mm}$ and $5.51 \mathrm{~mm}$ to $0.035 \mathrm{~mm}, 0.024 \mathrm{~mm}$ and $0.046 \mathrm{~mm}$. This proves the validity of the models proposed in this work.

\section{Conclusion:}

It is necessary to calibrate theodolites to fulfil the requirements of their use in robot metrology. This paper describes a simplified technique proposed for theodolite calibration using sets of vertical observation lines of known lengths. In this technique, the theodolites are regarded as $2 \mathrm{R}$ open-ended mechanisms with the endeffector axes directed along the line of sight. 
Mathematical models were developed using a non-singular kinematic representation and coded in a computer program, which was then employed successfully in program designed to simulate the calibration process. The simulation results indicate the suitability of the proposed technique for theodolite calibration applications.

\section{Appendix: The $\phi$-model}

The kinematic aspects of the $\phi$-model notation are shown in figure (8). The model is established by introducing an intermediate Cartesian system between the joint-frames number $i$ and $i+1$. The Z-axis of the new frame, which is referred to as the $\phi_{i}$-frame, lies in a plane parallel to the $\mathrm{X}_{i} \mathrm{Y}_{i}$-plane and at a distance, $d_{i}$, equal to the linear jointdisplacement from it. In case of a rotary joint, $d_{i}$ may be set equal to zero. This Zaxis, which may be referred to as $Z_{\phi_{i}}$, is initially set by the user at a constant angle, $\phi_{i}$, from the $\mathrm{X}_{i}$-axis. $\phi_{i}$, which is measured in a right-handed sense about $\mathrm{Z}_{i}$, is selected to ensure that $\mathrm{Z}_{\phi_{i}}$ may not be parallel to $\mathrm{Z}_{i+1}$. The $\mathrm{X}_{\phi_{i}}$-axis of the $\phi_{i}$-frame is then established in a plane perpendicular to both $\mathrm{Z}_{\phi_{i}}$ and $\mathrm{Z}_{i}$. The $\phi_{i}$-frame is then used to establish a Cartesian system, $\mathrm{X}_{i+1} \mathrm{Y}_{i+1} \mathrm{Z}_{i+1}$, about the $\mathrm{Z}_{i+1}$-axis in a DH-fashion. The $\phi_{i}$-frame and the $(i+1)$-frame are on the same rigid link and perform the same displacement $\left(d_{i}\right.$ or $\left.\theta_{i}\right)$ along or about the $Z_{i}$ respectively.

The transformation, ${ }_{i} \mathbf{T}^{i+1}$, from the (i+1)-frame to the i-frame may now be expressed as follows,

$$
{ }_{i} \mathbf{T}^{i+1}={ }_{i} \mathbf{T}^{\phi_{i}}{ }_{\phi_{i}} \mathbf{T}^{i+1}
$$


where ${ }_{i} \mathbf{T}^{\phi_{i}}$ and $\phi_{i} \mathbf{T}^{i+1}$ represent the transformation from the $\phi_{i}$-frame to the $i$-frame and from the $(i+1)$-frame to the $\phi_{i}$-frame respectively. These matrices may be expressed as follows,

$$
{ }_{i} \mathbf{T}^{\phi_{i}}=\left[\begin{array}{cccc}
-\sin \left(\phi_{i}+\theta_{i}\right) & 0 & \cos \left(\phi_{i}+\theta_{i}\right) & 0 \\
\cos \left(\phi_{i}+\theta_{i}\right) & 0 & \sin \left(\phi_{i}+\theta_{i}\right) & 0 \\
0 & 1 & 0 & d_{i} \\
0 & 0 & 0 & 1
\end{array}\right]
$$

and

$$
{ }_{\phi_{i}} \mathbf{T}^{i+1}=\left[\begin{array}{cccc}
\cos \left(\beta_{i}\right) & -\sin \left(\beta_{i}\right) \cos \left(\alpha_{i}\right) & \sin \left(\beta_{i}\right) \sin \left(\alpha_{i}\right) & b_{i} \cos \left(\beta_{i}\right) \\
\sin \left(\beta_{i}\right) & \cos \left(\beta_{i}\right) \cos \left(\alpha_{i}\right) & -\cos \left(\beta_{i}\right) \sin \left(\alpha_{i}\right) & b_{i} \sin \left(\beta_{i}\right) \\
0 & \sin \left(\alpha_{i}\right) & \cos \left(\alpha_{i}\right) & a_{i} \\
0 & 0 & 0 & 1
\end{array}\right]
$$

where $a_{i}, b_{i}, \alpha_{i}$ and $\beta_{i}$ are the DH-parameters which relate the (i+1)-frame to the $\phi_{i}$ frame as shown in figure (5). As the above expression for ${ }_{i} \mathbf{T}_{i}$ indicates, the angle between the $\mathrm{X}_{i}$ - and the $\mathrm{Z}_{\phi_{i}}$-axes is initially $\phi_{i}$. However with the onset of the rotational motion, this angle would vary by the value of the motor displacement, $\theta_{i}$. The expression also reveals that the $\phi_{i}$-frame may slide along the $\mathrm{Z}_{i}$-axis a distance $d_{i}$ if the joint was of the sliding type; in such a case $\theta_{i}$ may be set equal to zero.

\section{References:}

1) Bay, J. S., “Autonomous Parameter Identification by Optimal Learning Control,” IEEE J Control Systems, 1993, pp. 56-61. 
2) Driels, M. R. and Pathre, U. S., "Vision-Based Automatic Theodolite for Robot Calibration,” IEEE Trans. on Robotics and Automation, Vol. 7, No. 3, June 1991, pp. 351-360.

3) Everett, L. J. and Ives, T. W., "A Sensor Used for Measurements in the Calibration of Production Robots,” IEEE Trans., on Robotics and Automation, Vol. 12, No. 1, February 1996, pp. 121-125.

4) Everett, L. J., "Research Topics in Robot Calibration,” Robot Calibration. Edited by Bernhardt, R. and Albright, S. L. Chapman \& Hall, London, 1993.

5) Hartley, R. and Zissermann, A., "Multiple View Geometry in Computer Vision,” Cambridge University Press, 2000.

6) Hayati, S., “Robot Arm Geometric Link Parameter Estimation,” In Proceedings of $22^{\text {nd }}$ IEEE Conf. on Decision and Control, Dec. 1983, pp. 1477-1483.

7) Jarvis, J. F., “Calibration of Theodolites,” In Proceedings of IEEE Int. Conf. on Robotics and Automation, 1988, pp. 952-954.

8) Judd, R. P and Knasinski, A. B., “A Technique to Calibrate Industrial Robots with Experimental Verification,” IEEE Trans. on Robotics and Automation, Vol. 6, No. 1, Feb. 1990, pp. 20-30.

9) Legnani, J., Mina, C. and Trevelyan, J., "Static Calibration of Industrial Manipulators: Design of an Optical Instrumentation and Application to Scara Robot,” J. Robotics Systems, July 1996, pp. 445-460. 
10) Marquardt, D. W., “An Algorithm for Least-Squares Estimation of Nonlinear Parameters,” J. Soc. Industrial Appl. Math., Vol. 11, No. 2, June 1963, pp. 431441.

11) Mooring, B. W., Roth, Z. S. and Driels, M. R., "Fundamentals of Manipulator Calibration,” John Wiley \& Sons, New York, 1991.

12) Nakamura, O., Goto, M., Toyda, K., Takai, N., Kurosawa, T. and Nakamata, T., “A Laser Tracking Robot-Performance Calibration System Using a Ball-Seated Bearing Mechanisms and a Spherically Shaped Cat's-Eye Retroreflector,” Rev. Sc. Instrum., 65 (4), April 1994, pp. 1006-1011.

13) Preising, B. and Hsia, T. C., "Robot Performance Measurement and Calibration Using a 3D Camera Vision System,” In Proceedings of IEEE Int. Conf. on Robotics and Automation, Sacramento, April 1991, pp. 2079-2084.

14) Stone, H. W. and Sanderson, A. C., “A Prototype Arm Signature Identification System,” Proceedings of IEEE Int. Conf. on Robotics and Automation, Raleigh, 1987, pp. 175-182.

15) Sultan, I. A. and Wager, J G, “User-controlled kinematic modelling,” Int. J. Advanced Robotics, 1999, Vol 12, No. 6.

16) Sultan, I. A., “On the Positioning of Revolute-Joint Manipulators,” Int. J. of Robotic Systems, Vol. 17, No. 8, August 2000, pp 429-438.

17) Sultan, I. A. and Wager, J. G., “A Technique for the Independent-axis Calibration of Robot Manipulators with Experimental Verification,” Int. J. of Computer Integrated Manufacturing, Vol 14. No 4, July 2001. 
18) Tang, G. and Liu, L., "Robot Calibration Based on a Single Laser Displacement Meter,” Mechatronics, Vol. 3, 1993.

19) Vincze, M., Prenninger, J. P. and Gander H., “A Laser Tracking System to Measure Position and Orientation of Robot End Effectors Under Motion,” The Int. J Robotic Research, Vol. 13, No. 4, August 1994, pp. 305-314.

20) Whitney, D. E., Lozinski, C. A. and Rourke, J. M., "Industrial Robot Forward Calibration Method and Results,” ASME Journal of Dynamic Systems, Measurement and Control, Vol. 108, March 1986, pp 1-8.

21) Xu., Gang, "Unification of Stereo, Motion and Object Recognition via Epipolar Geometry,” $2^{\text {nd }}$ Int. Asian Conf. on Computer Vision, Singapore, 1995.

22) Xu, G. and Zhang, Z., "Epipolar Geometry in Stereo, Motion and Object Recognition- A Unified Approach,” Kluwer Academic Press, 1996.

23) Zhang, Z., “Determining the Epipolar Geometry and its Uncertainty: A Review,” Int. J. Computer Vision, Vol. 27, No. 2, pp 161-195, March 1998.

24) Zissermann, A. and Maybank, S. J., “A Case Against Epipolar Geometry,” $2^{\text {nd }}$ Int. Europe-US Workshop on Invariance, Ponta Delgada, Azores, Oct 1993. 


\section{List of Figures}

Figure (1): $\phi$-Model Assignment for a Two-Theodolite Module.

Figure (2): Block Diagram of the Simulation Procedure.

Figure (3): A Histogram of Typical Random Errors Added to Angular Measurements.

Figure (4): Block Diagram of the Performance Analysis Procedure.

Figure (5): The Effect of the Calibration Processes on the x-reading of Theodolites.

Figure (6): The Effect of the Calibration Processes on the y-reading of Theodolites.

Figure (7): The Effect of the Calibration Processes on the z-reading of Theodolites.

Figure (8): The Kinematic Notation of the $\phi$-Model.

\section{List of Tables}

Table (1): Nominal Values of System Parameters.

Table (2): Intentionally Deviated Values of System Parameters.

Table (3): Calibrated Values of System Parameters. 


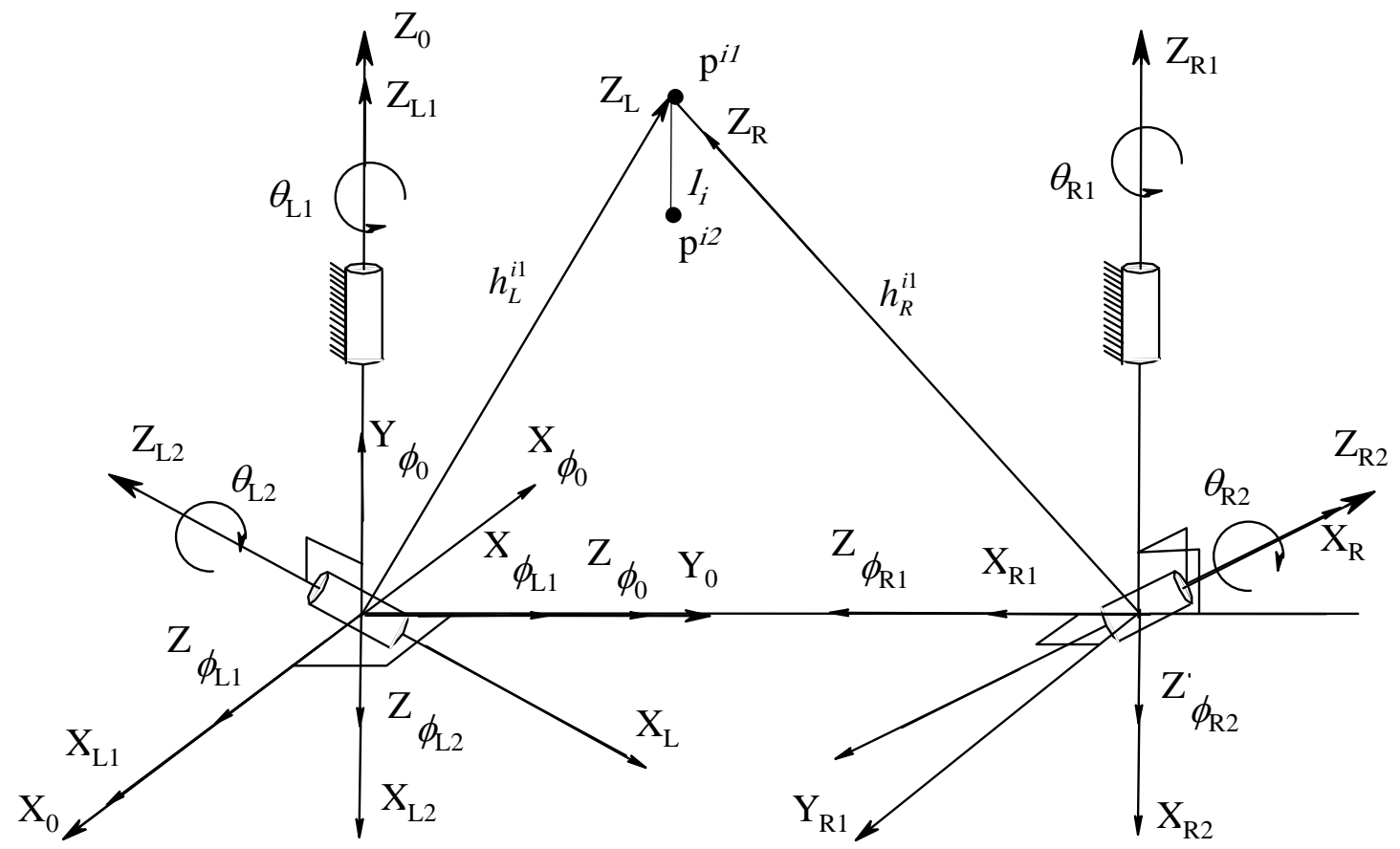

LHS Theodolite RHS Theodolite

Figure (1): $\phi$-Model Assignment for a Two-Theodolite Module. 


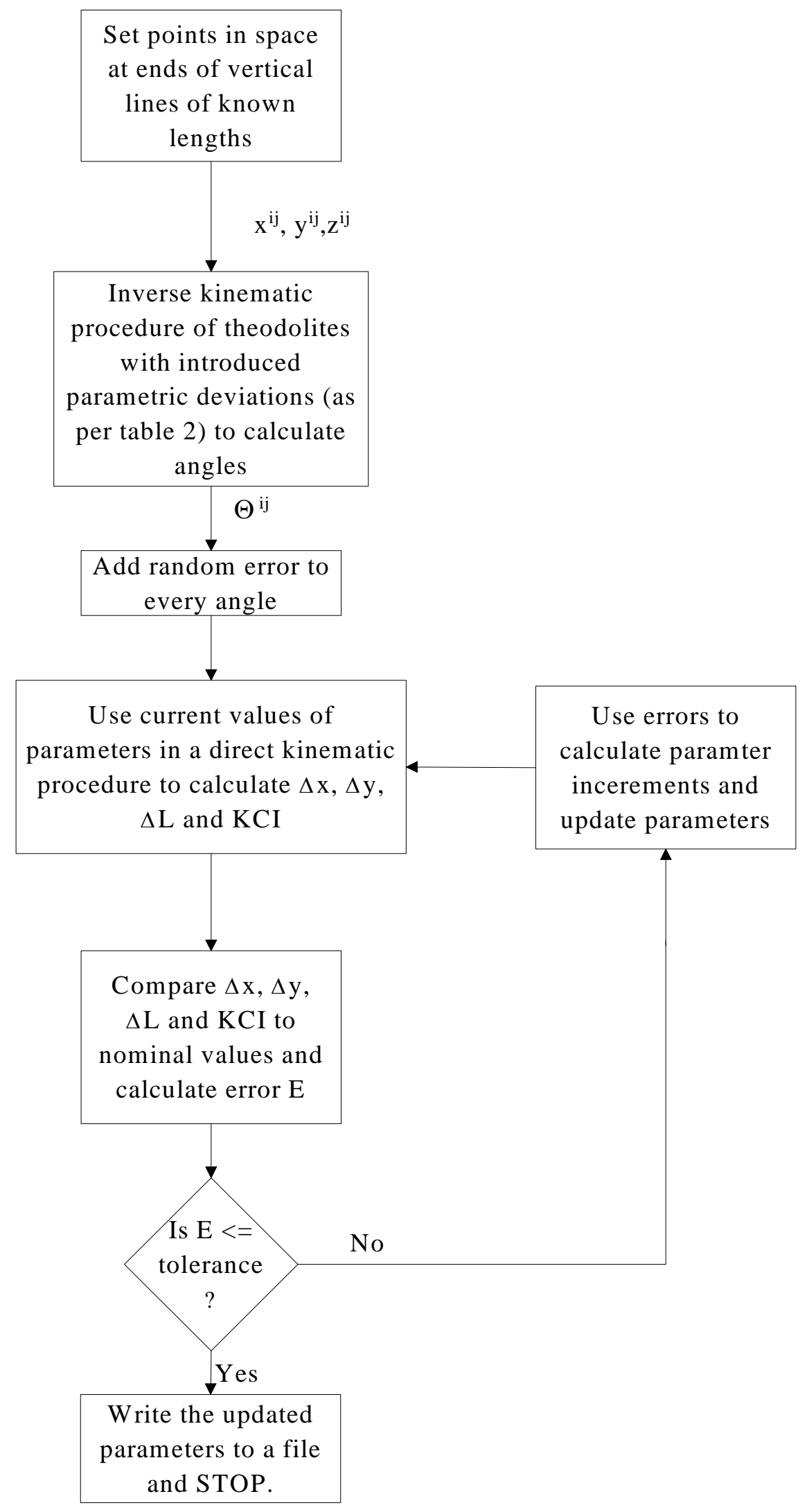

Figure (2): Block Diagram of the Simulation Procedure. 


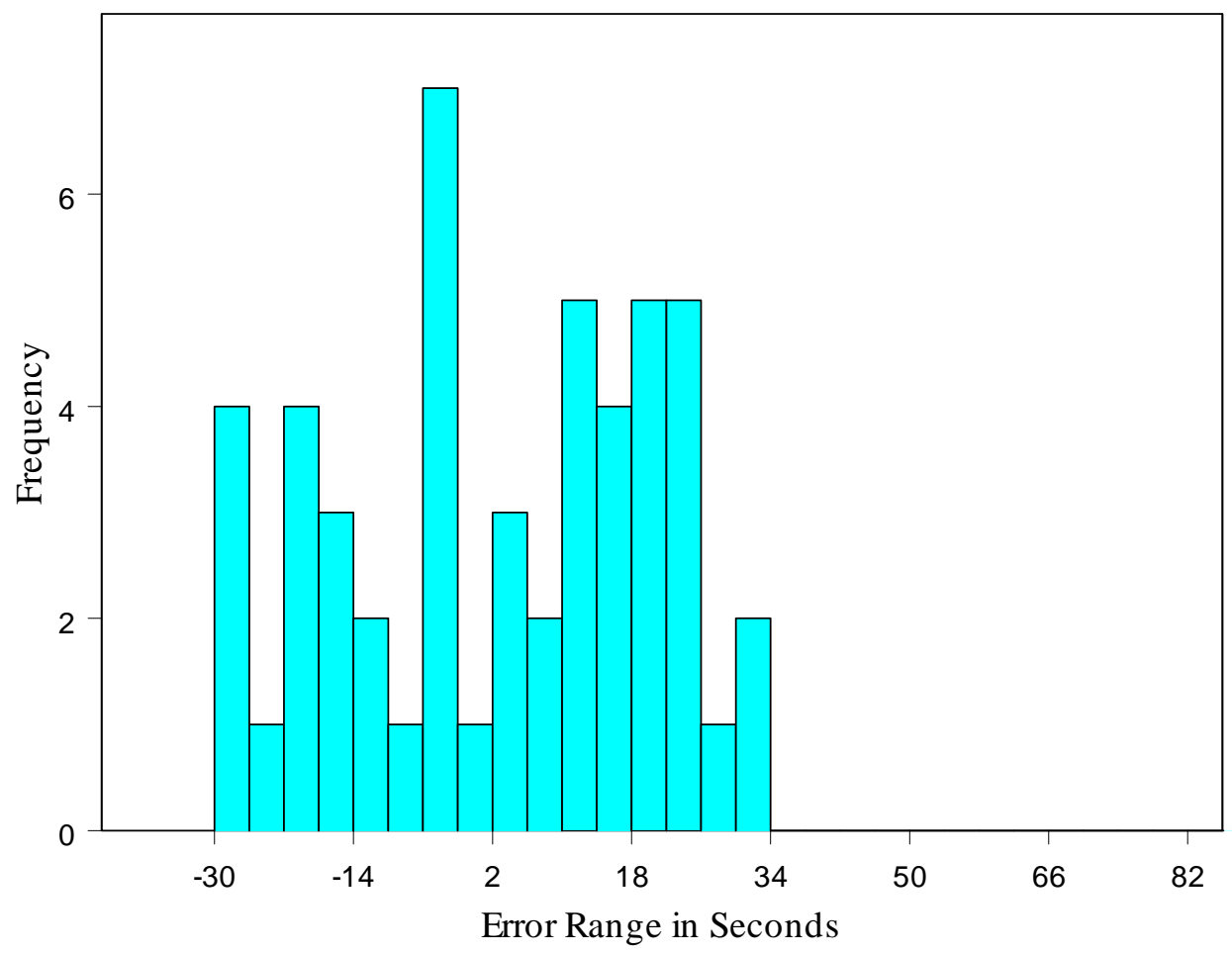

Figure (3): A Histogram of Typical Random Errors Added to Angular Measurements. 


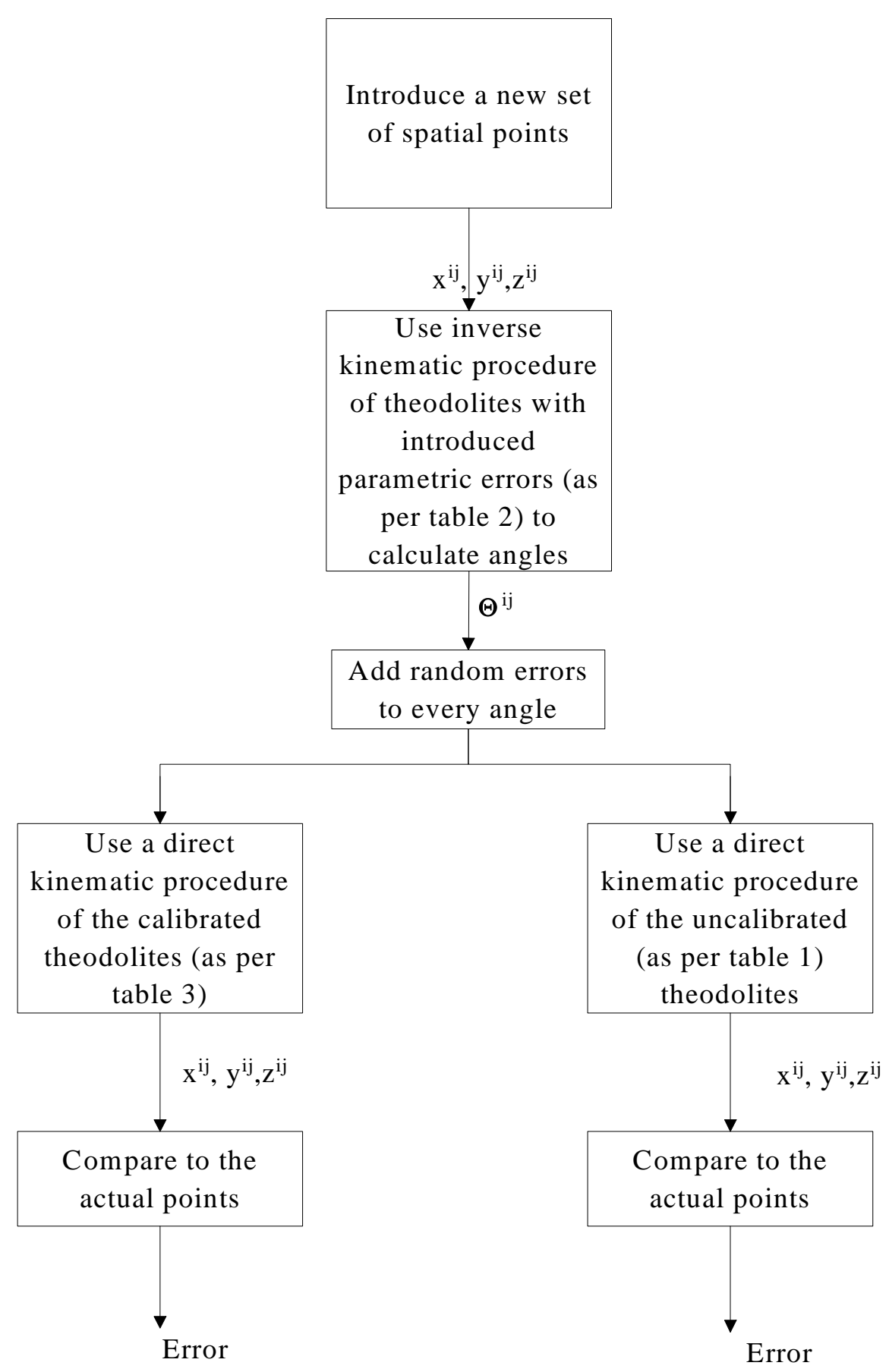

Figure (4): Block Diagram of the Performance Analysis Procedure. 


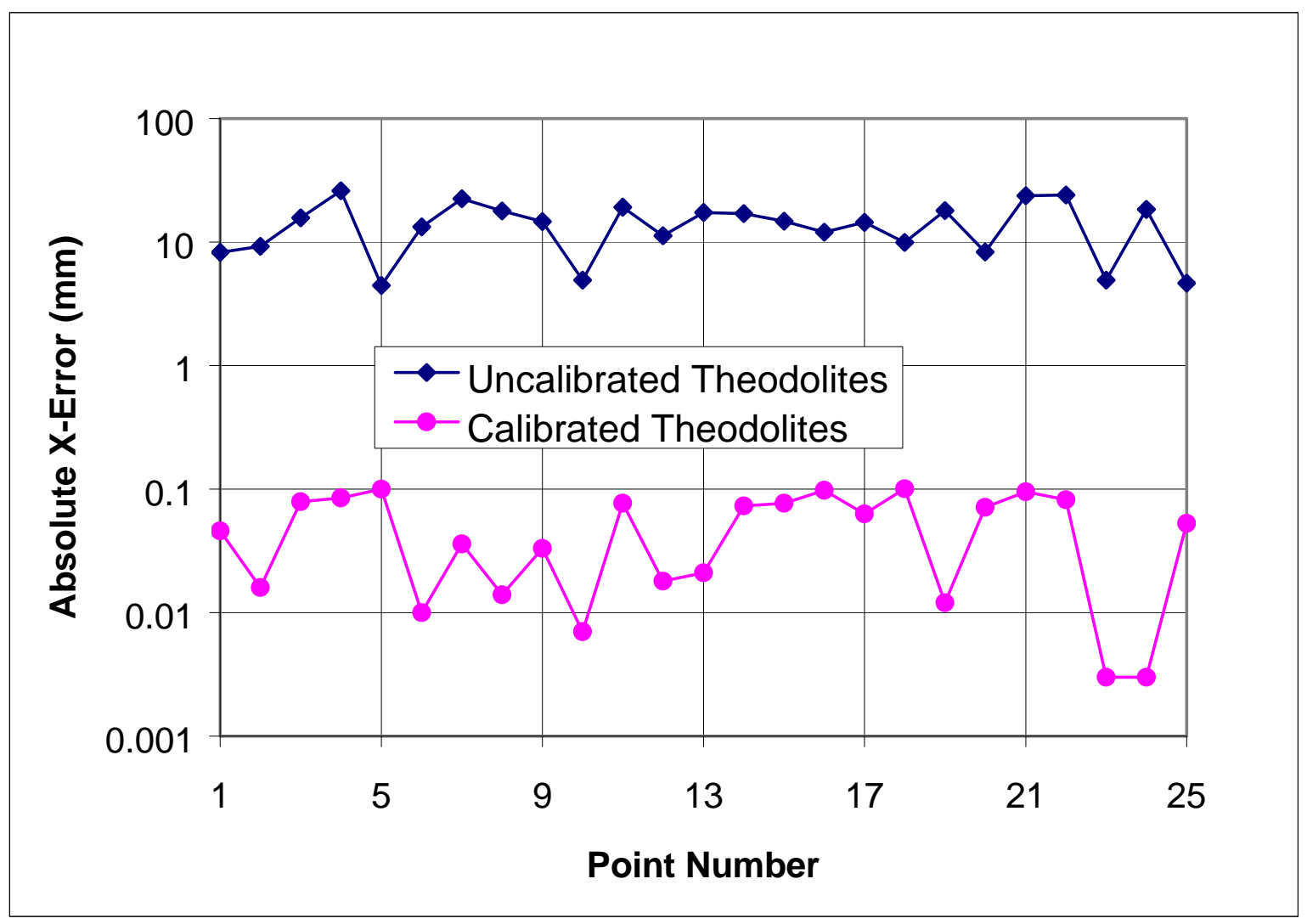

Figure (5): The Effect of the Calibration Processes on the X-reading of Theodolites. 


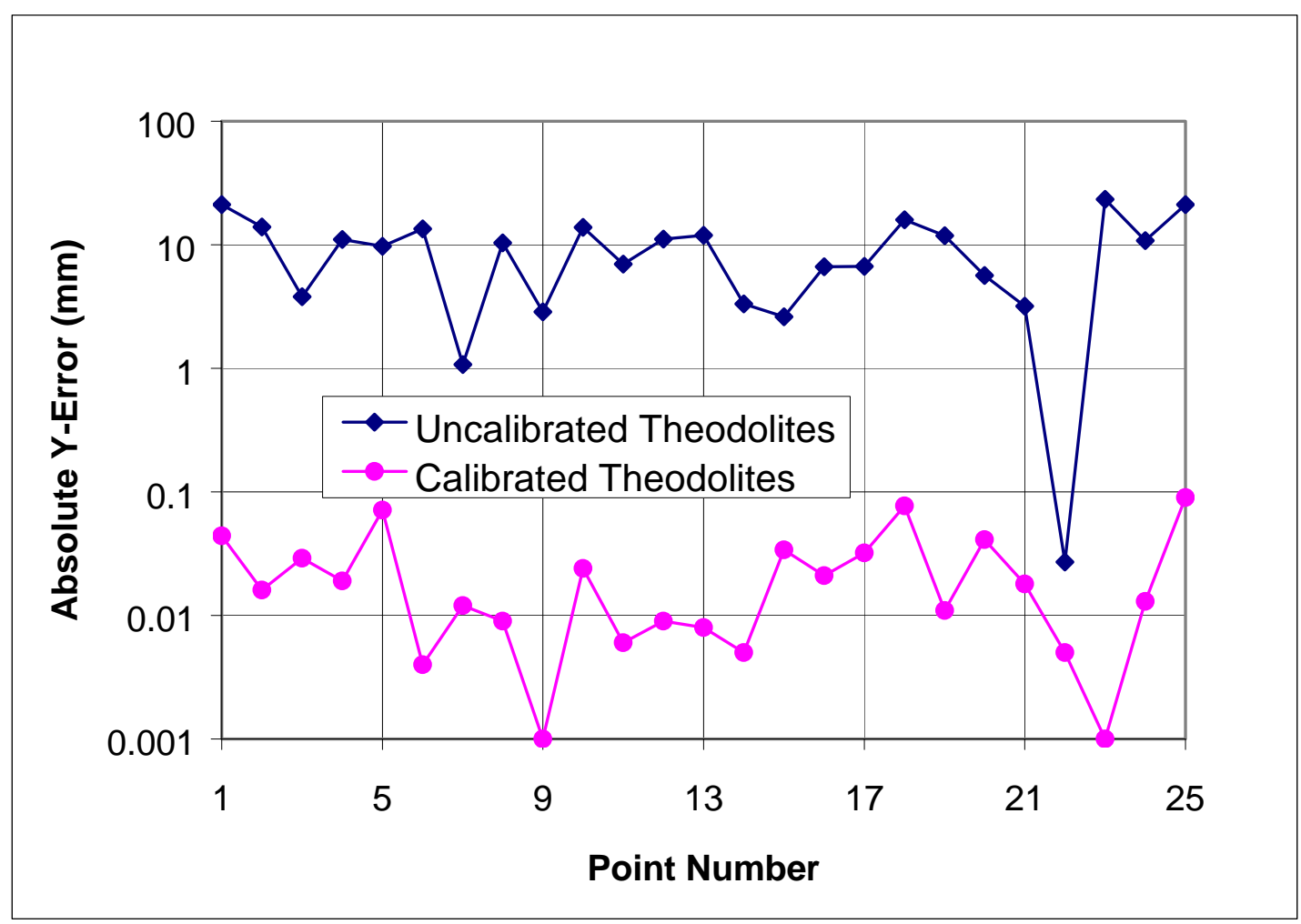

Figure (6): The Effect of the Calibration Processes on the Y-reading of Theodolites. 


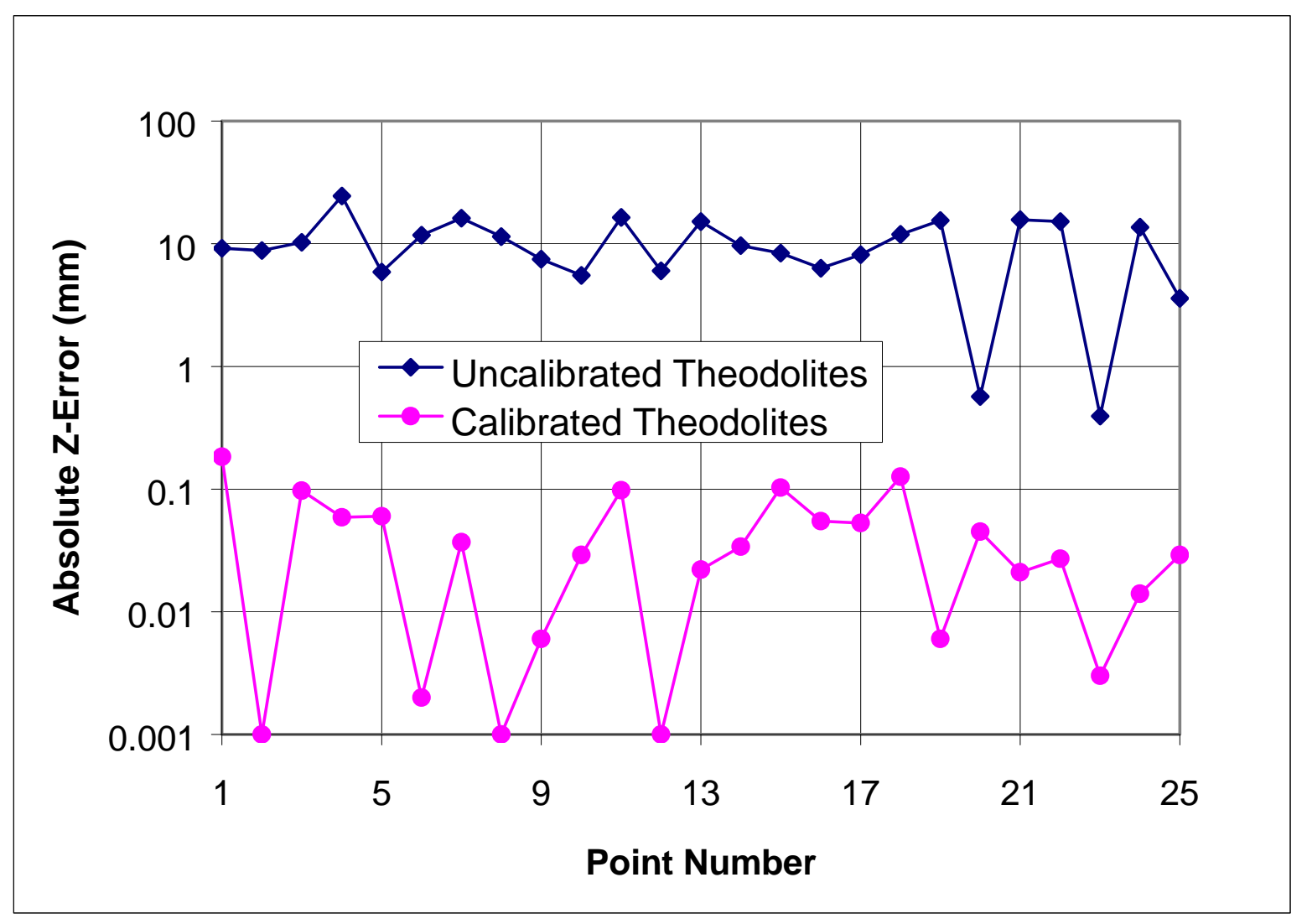

Figure (7): The Effect of the Calibration Processes on the Z-reading of Theodolites. 


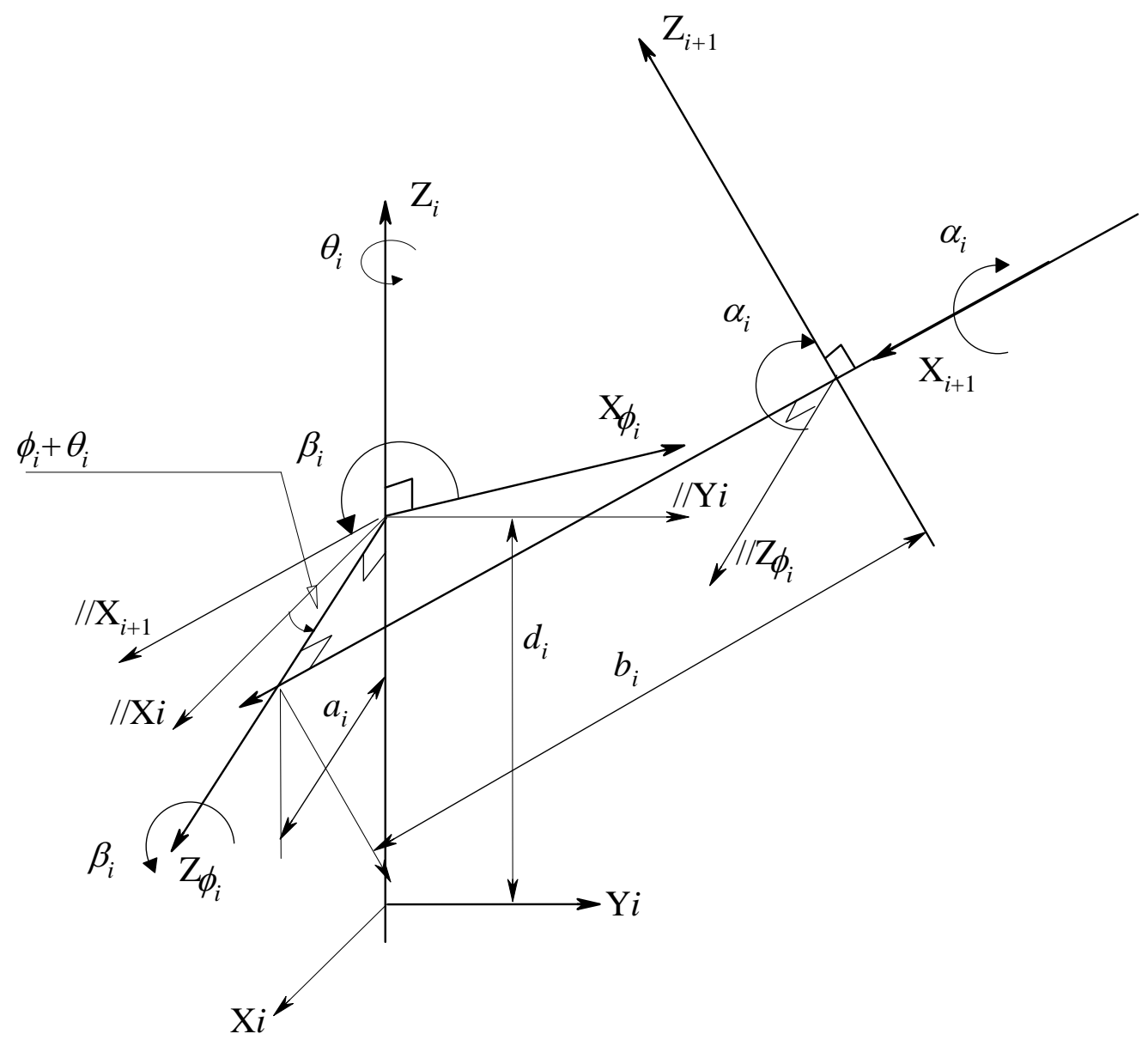

Figure (8): The Kinematic Notation of the $\phi$-Model. 
Table (1): Nominal Values of System Parameters.

\begin{tabular}{||l|c|c|c|c|c|c||}
\cline { 2 - 7 } \multicolumn{1}{c|}{} & 0 & $L 1$ & $L 2$ & $R 0$ & $R 2$ & $R 2$ \\
\hline \hline$a$ & 0.0 & 0.0 & 0.0 & 2400 & 0.0 & 0.0 \\
$b$ & 0.0 & 0.0 & 0.0 & 0.0 & 0.0 & 0.0 \\
$\alpha$ & $\pi / 2$ & $\pi / 2$ & $\pi / 2$ & $\pi / 2$ & $\pi / 2$ & $\pi / 2$ \\
$\beta$ & $\pi$ & $\pi / 2$ & $-\pi / 2$ & $\pi$ & $\pi / 2$ & $-\pi / 2$ \\
\hline
\end{tabular}

Table (2): Intentionally Deviated Values of System Parameters.

\begin{tabular}{||c|c|c|c|c|c|c||}
\cline { 2 - 7 } \multicolumn{1}{c|}{} & 0 & $L 1$ & $L 2$ & $R 0$ & $R 1$ & $R 2$ \\
\hline \hline$a$ & 0.0 & 0.229008 & -0.039649 & 2405.0 & 0.129916 & -0.385445 \\
$\alpha$ & 0.0 & 0.029187 & 0.199963 & 0.0 & -0.202099 & 0.102801 \\
$\beta$ & 1.580796 & 1.55074 & 1.630808 & 1.580786 & 1.582881 & 1.596907 \\
$\beta$ & 3.162 & 1.560002 & -1.530751 & 3.154 & 1.517744 & -1.584155 \\
\hline
\end{tabular}

Table (3): Calibrated Values of System Parameters.

\begin{tabular}{|l|c|c|c|c|c|c||}
\cline { 2 - 7 } \multicolumn{1}{c|}{} & 0 & $L 1$ & $L 2$ & $R 0$ & $R 1$ & $R 2$ \\
\hline \hline$a$ & 0.0 & 0.20266 & -0.03126 & 2405.017 & 0.13301 & -0.44304 \\
$\alpha$ & 0.0 & 0.1064 & 0.23157 & 0.0 & -0.05699 & 0.06682 \\
$\beta$ & 1.5807 & 1.5507 & 1.63081 & 1.58079 & 1.582889 & 1.59690 \\
$\beta$ & 3.1616 & 1.5600 & -1.53076 & 3.15359 & 1.517747 & -1.58415 \\
\hline
\end{tabular}

